\title{
Maintenance treatments for bipolar disorders
}

\author{
David I Pyle, Mood Disorders Unit Registrar, Prince of Wales Hospital, and Philip B \\ Mitchell, Professor and Head, School of Psychiatry, University of New South Wales, and \\ Consultant Psychiatrist, Prince of Wales Hospital and Black Dog Institute, Sydney
}

\section{Summary}

\section{Bipolar disorders are disabling and, for most} patients, recurrent illnesses. Lithium is the 'gold standard' mood stabiliser in terms of efficacy, but many patients find it difficult to tolerate. The anticonvulsants sodium valproate and carbamazepine are useful despite minimal controlled evidence for their prophylactic efficacy. The approval of olanzapine and lamotrigine for maintenance treatment increases the choice of drug therapy. These new drugs, in conjunction with the development of effective psychological interventions, mean that the clinician has an increasing range of effective options to offer patients with these disabling and challenging conditions.

Key words: carbamazepine, lithium, lamotrigine, olanzapine, sodium valproate.

(Aust Prescr 2007;30:70-3)

\section{Introduction}

Bipolar disorders (see box) are relatively common conditions with a lifetime prevalence of up to $4 \% .^{1}$ They lead to levels of disability which are greater than those associated with major depressive disorder (unipolar depression). ${ }^{2}$ Rates of disrupted relationships are high and many sufferers are unemployed and in receipt of government benefits. At least a quarter have a history of suicide attempts, with $10-20 \%$ of all patients ending their life by their own hand.

While effective and rapid management of acute episodes of mania and bipolar depression are critical components of treatment, the prevention of relapse is probably the most important aspect of management. Bipolar disorders are highly recurrent for most patients. It is the recurring nature of the condition that, unless adequately treated, gradually takes its toll in terms of the patient's capacity to maintain relationships, career and self-esteem. The average patient experiences a major relapse every 17 to 30 months, with episodes frequently lasting between three and six months. At least $25 \%$ will go through phases of rapid-cycling illness in which they experience at least four episodes in a year. ${ }^{3}$
The challenge for the treating clinician - be that a general practitioner ${ }^{4}$ or psychiatrist - is to ensure adequate long-term control of the illness. Effective maintenance treatment can make an enormous difference to the lives of those with bipolar disorders. The benefits observed can be some of the most dramatic seen in medical practice.

\section{Which patients should be commenced on maintenance treatment?}

There are different guidelines, but the basic principle is that most patients with recurrent, severe or disabling illness are highly likely to benefit from prophylactic treatment. Usually (but not always) the maintenance treatment will be a continuation of the drug that was effective for acute treatment (Table 1). Some of these drugs are currently not subsidised for maintenance treatment (Table 2).

\section{Lithium}

Although lithium was first discovered to be effective in mania in 1949, by the Melbourne psychiatrist John Cade, it is still the 'gold standard' therapy. Despite the intervening 58 years, no treatments of greater potency have yet been developed.

Many patients are unable to tolerate lithium and it has limited effectiveness for the depressive phase of bipolar disorders.

$\begin{array}{ll}\text { Bipolar I disorder } & \begin{array}{l}\text { At least one episode of mania (current or } \\ \text { past) } \\ \text { Usually (but not necessarily) episodes of } \\ \text { depression }\end{array} \\ \text { Bipolar II disorder } & \text { Episodes of hypomania and depression } \\ & \text { No manic episodes } \\ \text { Mania } & \begin{array}{l}\text { Pathologically elevated or euphoric } \\ \text { mood (often also irritable) lasting at least } \\ \end{array} \\ & \text { one week. There is evidence of marked } \\ & \text { impairment of functioning. Delusions } \\ & \text { hospitalisation may be required. } \\ \text { Hypomania } & \begin{array}{l}\text { Pathologically elevated (or irritable) mood } \\ \text { lasting at least 2-4 days. While mood } \\ \text { and behaviour are distinctly different } \\ \text { from normal, functioning is not severely } \\ \text { impaired. Psychotic features do not occur } \\ \text { and hospitalisation is unnecessary. }\end{array}\end{array}$


Table 1

Relative efficacy of drugs in preventing manic and depressed episodes

\begin{tabular}{llc}
\hline & \multicolumn{2}{c}{ Preventive potency } \\
\cline { 2 - 3 } & Mania & Depression \\
\hline Lithium & ++ & + \\
Carbamazepine & + & + \\
Valproate & + & + \\
Lamotrigine & +- & ++ \\
Olanzapine & $++{ }^{*}$ & + \\
\hline
\end{tabular}

++ strong evidence

+ reasonable evidence

+ - equivocal evidence

* one (unreplicated) study demonstrated superiority to lithium for prophylaxis in mania

There are more positive randomised double-blind controlled trials for lithium as a maintenance therapy than for any other treatment. Several meta-analyses have confirmed the efficacy of lithium, particularly in preventing manic relapse. ${ }^{5}$ Its capacity to prevent depressive relapse is less clear-cut. Consequently, many patients on lithium suffer from frequent and prolonged depressive episodes, despite dramatic suppression of the periods of elevated mood. Non-compliance is common $(20-50 \%$ of patients) and if lithium is abruptly discontinued, the chance of sudden relapse into mania is considerable.

The main drawbacks of lithium are the need for serum concentration monitoring, the possibility of serious toxicity, and the risk of thyroid (and less commonly renal) impairment. Tremors, increased muscle tone, hyperreflexia and disorientation are signs of severe toxicity.

\section{Anticonvulsants}

In Australia sodium valproate is an anticonvulsant drug that is approved for acute treatment of mania. It is also commonly used as an alternative to lithium for maintenance treatment of bipolar disorders. Carbamazepine, another anticonvulsant, is approved for the management of mania and the maintenance treatment of bipolar disorder.

The only placebo-controlled trial of carbamazepine in prophylaxis failed to show superiority over placebo. However, most of the five randomised double-blind comparisons with lithium reported no difference between lithium and carbamazepine. There has been only one double-blind trial of sodium valproate in the prophylaxis of bipolar disorders. This found no differences between either valproate or lithium when compared to placebo. ${ }^{6}$ Despite this lack of evidence from controlled trials, clinical experience worldwide has seemed to confirm the benefit of these drugs in reducing relapse rates.
Table 2

Status of drugs currently approved in Australia for bipolar disorders

\begin{tabular}{lcccc}
\hline & \multicolumn{2}{c}{ Marketing approval } & \multicolumn{2}{c}{ Subsidised indications } \\
\cline { 2 - 5 } & $\begin{array}{l}\text { Acute } \\
\text { mania Maintenance }\end{array}$ & $\begin{array}{l}\text { Acute } \\
\text { mania }\end{array}$ & Maintenance \\
\hline Lithium & $\checkmark$ & $\checkmark$ & $\checkmark$ & $\checkmark$ \\
Carbamazepine & $\checkmark$ & $\checkmark$ & $\checkmark$ & $\checkmark$ \\
Valproate & $\checkmark$ & $x$ & $\checkmark$ & $x$ \\
Lamotrigine & $x$ & $J^{*}$ & $x$ & $x$ \\
Olanzapine & $\checkmark$ & $\checkmark$ & $x$ & $\checkmark$ \\
Quetiapine & $\checkmark$ & $x$ & $x$ & $x$ \\
Risperidone & $\checkmark$ & $x$ & $x$ & $x$ \\
Ziprasidone & $\checkmark$ & $x$ & $x$ & $x$ \\
\hline
\end{tabular}

* Approved for prevention of episodes of bipolar depression only. This approval is not presently listed in the product information.

There is no drug or medicine specifically approved in Australia for the acute treatment of bipolar depression.

\section{Lamotrigine}

Lamotrigine is an anticonvulsant that may also be used in Australia for the prevention of bipolar depressive episodes. This indication is not subsidised by the Pharmaceutical Benefits Scheme (PBS). There is evidence from one placebocontrolled trial for the efficacy of lamotrigine in the acute treatment of bipolar depression, but this was not replicated in several subsequent trials. Lamotrigine is neither acutely nor prophylactically effective in unipolar depression. It is not significantly superior to placebo in the acute treatment of mania. In two trials of maintenance treatment involving 638 patients with bipolar I disorder over 18 months, lamotrigine was superior to placebo in the prevention of depressive episodes, while lithium was more effective than placebo in the prevention of mania. ${ }^{7}$ A pooled analysis of both studies showed that lamotrigine was more effective than placebo for preventing depression, and lithium was more effective for mania. It also showed that lamotrigine was statistically more effective than placebo in the prevention of manic episodes, but this appeared to be of limited clinical significance. ${ }^{8}$

The main safety problem with lamotrigine is serious rash. The development of Stevens-Johnson syndrome is a major concern as it may be fatal. Major risk factors for serious rash are rapid dose escalation and failure to reduce the dose of lamotrigine on co-administration with sodium valproate.

\section{Antipsychotics}

The antipsychotic olanzapine has been approved in Australia for prevention of relapse in bipolar I disorder and this indication is 
included in the PBS. Olanzapine is also approved for the acute treatment of mania.

The strongest evidence for the prophylactic efficacy of olanzapine comes from a 12-month randomised double-blind comparison with lithium. ${ }^{9}$ Olanzapine was superior to lithium in the prevention of manic and mixed episodes and equivalent to lithium for reducing bipolar depressive episodes even in the absence of psychosis. As yet, no other studies have confirmed that olanzapine has greater efficacy than lithium in preventing manic relapse.

At present there are few reports about the long-term preventive efficacy of other atypical antipsychotics, although the effect of olanzapine may turn out to be a class effect. Risperidone has been approved in Australia for continuation for six months following acute treatment of mania.

The major safety concerns with olanzapine and some other atypical antipsychotics are substantial weight gain, hyperlipidaemia and diabetes. During long-term treatment with olanzapine, lipids and glucose should be monitored, and active means instituted to encourage diet and exercise.

\section{Combination therapy}

There is minimal evidence to support the use of combinations of drugs for maintenance treatment. The main evidence comes from a study in the 1990s which found that patients unresponsive to monotherapy with lithium or anticonvulsants often responded to combined therapies. The effective combinations were lithium and carbamazepine, and lithium and valproate. $^{10}$

\section{Is there a role for long-term antidepressants?}

For many patients, the episodes of mania are relatively easily treated, but depressive episodes are frequently less amenable to treatment. There is currently considerable controversy internationally over adding long-term antidepressants to the maintenance treatment of bipolar disorders. Antidepressants may induce manic episodes or even a rapid-cycling pattern, but the frequency of this is debated as there is some evidence that suggests induction of mania is relatively uncommon. There is some evidence that continuing antidepressants in patients who respond acutely to them has a prophylactic benefit. In one study $70 \%$ of the patients who stopped their antidepressants early relapsed into depression, compared to $36 \%$ of the patients who continued their antidepressants. ${ }^{11}$ Some (particularly US) authorities argue that antidepressants should rarely be used in long-term treatment.

\section{Psychological interventions}

Strong evidence for the benefits of psychological interventions in reducing the likelihood of relapse (particularly depressive episodes) is accumulating from a series of randomised controlled trials. Educational techniques, empowering the patient to take responsibility for the management of their illness, have been shown to reduce relapse and improve social functioning and employment. Cognitive therapy is aimed at improving skills in managing stress and symptoms, and in identifying early warning signs of impending relapse, and teaching skills to challenge and alter unhelpful thinking styles. ${ }^{12}$ It improves mood, coping and adherence, and reduces recurrence. ${ }^{13}$ Interpersonal and social rhythm therapy teaches patients to regulate their social habits, sleep patterns and daily routines at times of stress. ${ }^{14}$

\section{Conclusion}

New treatments, in conjunction with the development of effective psychological interventions for bipolar disorders, mean that the clinician has an increasing range of effective maintenance therapies to offer patients with these disabling and challenging conditions. While none of the newer drugs has been shown to be more effective than lithium, they are better tolerated by some patients.

\section{References}

1. Kessler RC, Berglund P, Demmler O, Jin R, Merikangas KR, Walters EE. Lifetime prevalence and age-of-onset distributions of DSM-IV disorders in the National Comorbidity Survey Replication. Arch Gen Psychiatry 2005;62:593-602.

2. Mitchell PB, SladeT, Andrews G. Twelve-month prevalence and disability of DSM-IV bipolar disorder in an Australian general population survey. Psychol Med 2004;34:777-85.

3. Angst J, Sallero R. Historical perspectives and natural history of bipolar disorder. Biol Psychiatry 2000;48:445-57.

4. Mitchell PB, Ball JR, Best JA, Gould BM, Malhi GS, Riley GJ, et al. The management of bipolar disorder in general practice. Med J Aust 2006;184:566-70.

5. Geddes JR, Burgess S, Hawton K, Jamison K, Goodwin GM. Long-term lithium therapy for bipolar disorder: systematic review and meta-analysis of randomized controlled trials. Am J Psychiatry 2004;161:217-22.

6. Bowden CL, Calabrese JR, McElroy SL, Gyulai L, Wassef A, Petty $\mathrm{F}$, et al. A randomized, placebo-controlled 12-month trial of divalproex and lithium in treatment of outpatients with bipolar I disorder. Arch Gen Psychiatry 2000;57:481-9.

7. Bowden CL, Calabrese JR, Sachs G, Yatham LN, Asghar SA, Hompland M, et al. A placebo-controlled 18-month trial of lamotrigine and lithium maintenance treatment in recently manic or hypomanic patients with bipolar I disorder. Arch Gen Psychiatry 2003;60:392-400.

8. Goodwin GM, Bowden CL, Calabrese JR, Grunze H, Kasper S, White $\mathrm{R}$, et al. A pooled analysis of 2 placebo-controlled 18-month trials of lamotrigine and lithium maintenance in bipolar I disorder. J Clin Psychiatry 2004;65:432-41.

9. Tohen M, Greil W, Calabrese JR, Sachs GS, Yatham LN, Oerlingshausen BM, et al. Olanzapine versus lithium in the maintenance treatment of bipolar disorder: a 12-month, randomized, double-blind, controlled clinical trial. Am J Psychiatry 2005;162:1281-90. 
10. Denicoff KD, Smith-Jackson EE, Bryan AL, Ali SO, Post RM. Valproate prophylaxis in a prospective clinical trial of refractory bipolar disorder. Am J Psychiatry 1997;154:1456-8.

11. Altshuler L, Suppes T, Black D, Nolen WA, Keck PE, Frye MA, et al. Impact of antidepressant discontinuation after acute bipolar depression remission on rates of depressive relapse at 1-year follow-up. Am J Psychiatry 2003;160:1252-62.

12. Ball JR, Mitchell PB, Corry JC, Skillecorn A, Smith M, Malhi GS. A randomized controlled trial of cognitive therapy for bipolar disorder: focus on long-term change. J Clin Psychiatry 2006;67:277-86.

13. Scott J, Paykel E, Morriss R, Bentall R, Kinderman P, Johnson T, et al. Cognitive-behavioural therapy for severe and recurrent bipolar disorders: randomised controlled trial. Br J Psychiatry 2006;188:313-20.

14. Frank E, Kupfer DJ, Thase ME, Mallinger AG, Swartz HA, Fagiolini AM, et al. Two-year outcomes for interpersonal and social rhythm therapy in individuals with bipolar I disorder. Arch Gen Psychiatry 2005;62:996-1004.
Professor Mitchell has received honoraria from GlaxoSmithKline, Eli Lilly and AstraZeneca for lectures, and has served on an advisory board for Eli Lilly in the last three years.

\section{Self-test questions}

The following statements are either true or false (answers on page 83)

5. In bipolar disorders, lithium is more effective at preventing manic relapse than depressive relapse.

6. Adding an antidepressant to the maintenance treatment of bipolar disorders may induce mania.

\section{Book review}

Therapeutic Guidelines: Antibiotic. Version 13.

Melbourne: Therapeutic Guidelines Limited; 2006. 422 pages. Price $\$ 39$, students $\$ 30$, plus postage

\section{Sophie Dwyer, Academic General Practice registrar, Discipline of General Practice, University of Adelaide}

Therapeutic Guidelines: Antibiotic is the original and most widely distributed book in the Therapeutic Guidelines series. There have been revisions and additions to the content, but there have been few changes to the concise and easy-to-use format of this book.

The primary use of Therapeutic Guidelines: Antibiotic is as a quick evidence-based reference guide for practitioners in selecting an appropriate antibiotic. The succinct discussion relevant to clinical diagnosis and common organisms is as valuable as the actual recommendations. Where the use of antibiotics is controversial or not indicated for a particular condition, this is discussed, as is non-pharmacologic management. Importantly for infectious diseases, the content is distinctly Australian.

The book commences with a discussion of the principles of antimicrobial use that covers basics such as antibiotic choice, duration of treatment and resistance. The 'Getting to know your drugs' chapter looks briefly at antimicrobials by class. Later chapters discuss administration routes, pregnancy and lactation with a detailed section on dose reduction in renal failure. Specific information on particular drugs is better covered by books such as the Australian Medicines Handbook.

The largest component of the book is arranged by system with conditions ordered alphabetically. Recommendations for firstline antimicrobial treatment are generally accompanied by at least one alternative. Chapters are devoted to specific infections such as malaria, HIV and mycobacteria. A whole chapter is now dedicated to the management of pneumonia. The chapter on the management of severe sepsis has been expanded and includes more information on initial management than the previous version. Newly included treatment algorithms cover important conditions such as pneumonia and meningitis. The rationale for medical and surgical antibiotic prophylaxis is also covered.

This guide is a well entrenched source of reliable information for general practitioners, hospital staff and specialists. The pocket-sized book is also available in 'updateable' versions for desktop computers and personal digital assistants (PDAs) which means many practitioners have several avenues to access this information. These electronic versions have the advantage of including all the titles in the Therapeutic Guidelines series. 